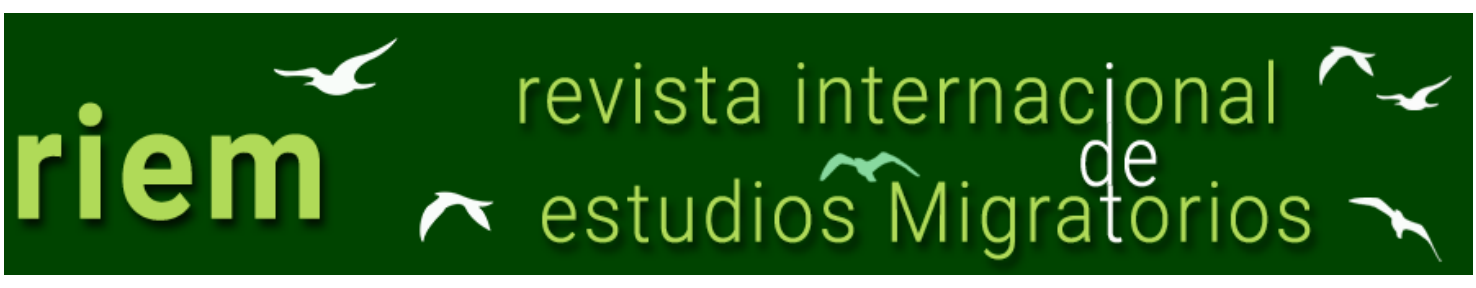

ISSN: $2173-1950$

\title{
Relación entre el proceso de aculturación y las actitudes hacia comportamientos sexuales en personas de origen marroquí: un estudio preliminar
}

Andrea Blanc Molina ${ }^{1}$

Resumen: El fenómeno de las migraciones internacionales y la influencia de la cultura en la sexualidad han conducido al desarrollo de estudios que relacionan la aculturación con las actitudes sexuales. Sin embargo, hasta la fecha, ningún estudio ha relacionado el proceso de aculturación con las actitudes sexuales en España. Por ello, el objetivo del estudio fue relacionar el proceso de aculturación con las actitudes hacia comportamientos sexuales en personas de origen marroquí en este país. Participaron un total de 110 personas de origen marroquí (51.8\% mujeres) entre 18 y 30 años. Se administró de forma online una escala de actitudes hacia comportamientos sexuales, dos escalas sobre preferencias de aculturación en los ámbitos más privados propuestos por el modelo ampliado de aculturación relativa (MAAR) e indicadores sobre preferencias y estrategias en el ámbito sexual. Los resultados muestran que en las mujeres existe relación negativa entre el mantenimiento de la cultura de origen en los ámbitos del MAAR y las actitudes hacia comportamientos sexuales y en los hombres existe relación positiva entre la adopción de la cultura de acogida y dichas actitudes. Los resultados también muestran que las preferencias de adopción y la adopción de las costumbres sexuales de la cultura española se relacionan positivamente con dichas actitudes. Estos resultados confirman la importancia del proceso de aculturación en las actitudes hacia comportamientos sexuales en las personas de origen marroquí en España.

Palabras Clave: actitudes hacia comportamientos sexuales, proceso de aculturación, marroquíes.

\footnotetext{
${ }^{1}$ Departamento de Psicología Clínica y Experimental, Universidad de Huelva, España. andrea.blanc@dpces.uhu.es
} 


\title{
Relationship between the acculturation process and the attitudes toward sexual behaviors in people of Moroccan origin: a preliminary study
}

\begin{abstract}
The phenomenon of international migrations and the influence of culture on sexuality have led to the development of studies that relate acculturation with sexual attitudes. However, to date, no study has related the acculturation process to sexual attitudes in Spain. Thus, the aim of the study was to relate the acculturation process to attitudes toward sexual behaviors in people of Moroccan origin in this country. A total of 110 people of Moroccan origin (51.8\% women) between 18 and 30 years old participated. A scale of attitudes toward sexual behaviors, two scales of acculturation preferences in the most private areas proposed by the relative acculturation extended model (RAEM), and indicators of preferences and strategies in the sexual area were administered online. The results show that in women there is a negative relationship between the maintenance of the origin culture in the RAEM areas and attitudes toward sexual behaviors, and in men there is a positive relationship between the adoption of the host culture and these attitudes. The results also show that adoption preferences and the adoption of sexual customs in Spanish culture are positively related to these attitudes. These results confirm the importance of the acculturation process in the attitudes toward sexual behaviors in people of Moroccan origin in Spain.
\end{abstract}

Keywords: attitudes towards sexual behaviors, acculturation process, Moroccan. 


\section{Introducción}

El papel relevante de la cultura en la sexualidad ha sido demostrado en la literatura científica (p.e., Du y Li, 2015). Este importante papel junto con el fenómeno de las migraciones internacionales ha conducido al desarrollo de estudios que relacionan el origen etnocultural con las actitudes sexuales (p.e., Ahrold y Meston, 2010). Los estudios muestran que en cada cultura la sexualidad es concebida de forma diferente, otorgándole distintos significados e importancia (Heinemann et al., 2016). Las diferentes formas de concebir la sexualidad en las distintas culturas conducen a que existan diferencias entre los distintos grupos etnoculturales en las actitudes sexuales (p.e., Eisenman y Dantzker, 2006; Fugère et al., 2008).

La influencia de la cultura en la sexualidad y el fenómeno de las migraciones internacionales también han conducido al desarrollo de estudios que relacionan la aculturación con las actitudes sexuales (p.e., Ahrold y Meston, 2010). La aculturación puede definirse como el proceso de cambio cultural y psicológico que se produce cuando hay un contacto continuo entre personas de diferentes grupos etnoculturales (Berry, 2003). De acuerdo con los modelos bidimensionales de aculturación (p.e., Navas et al., 2005) en dicho proceso se debe considerar de forma independiente el mantenimiento de las costumbres de la cultura de origen y la adopción de las costumbres de la cultura de acogida. En general, los estudios que han relacionado el proceso de aculturación con las actitudes sexuales han encontrado que la adopción de las costumbres de la cultura de acogida se relaciona de forma positiva con las actitudes sexuales (p.e., Ahrold y Meston, 2010; Blanc y Rojas, 2020). Las personas que adoptan más costumbres de la cultura de acogida tienen actitudes sexuales más positivas o liberales. Sin embargo, los resultados de la relación entre el mantenimiento de las costumbres de la cultura de origen y las actitudes sexuales no son tan consistentes (Dang et al., 2019). Por ejemplo, mientras que Ahrold y Meston (2010) encuentran que el mantenimiento de la cultura de origen se relaciona negativamente con las actitudes sexuales liberales, posteriormente Dang et al. (2019) encuentran una correlación positiva. Incluso en algunos estudios no se ha hallado ninguna relación significativa entre el mantenimiento de la cultura de origen y las actitudes sexuales (Brotto et al., 2007).

Por otro lado, algunos de los estudios que relacionan el proceso de aculturación con las actitudes sexuales han encontrado el efecto de variables moderadoras (p.e., Ahrold y Meston, 2010; Blanc y Rojas, 2020) o mediadoras (p.e., Woo et al., 2011). Por ejemplo, Blanc y Rojas (2020) encuentran que el sexo modera la relación entre el proceso de aculturación y las actitudes sexuales. Mientras que hay relación entre la adopción de la 
cultura dominante y las actitudes sexuales en hombres de un determinado grupo etnocultural, no ocurre lo mismo en el caso de las mujeres del mismo grupo. Estos autores también encuentran que el proceso de aculturación modera la relación entre el sexo y las actitudes sexuales. Mientras que en altos niveles de mantenimiento hay diferencias entre hombres y mujeres de un determinado grupo etnocultural en las actitudes sexuales, en bajos niveles de mantenimiento no se dan esas diferencias (Blanc y Rojas, 2020). Los estudios también muestran que el origen etnocultural modera la relación entre el proceso de aculturación y las actitudes sexuales (Ahrold y Meston, 2010; Blanc y Rojas, 2020). Esto es, las relaciones entre el proceso de aculturación y las actitudes sexuales no son igual en todos los grupos etnoculturales. En cuanto a los efectos de mediación, se ha encontrado que las actitudes sexuales median la relación entre el proceso de aculturación y la salud sexual (Woo et al., 2011).

Los estudios que han relacionado el proceso de aculturación y las actitudes sexuales no han sido muy abundantes en la literatura científica y se han desarrollado en Estados Unidos (p.e., Guo, 2019) y Canadá (p.e., Dang et al., 2019). Estos estudios se han centrado principalmente en asiáticos (p.e., Woo et al., 2011) y latinos (p.e., Castañeda, 2017), aunque algún estudio también ha incluido a africanos (Blanc y Rojas, 2020) o iraníes (p.e., Abdolsalehi-Najafi y Beckman, 2013). En España, hasta la fecha no existen estudios que hayan relacionado el proceso de aculturación con las actitudes sexuales. Por ello, en el presente estudio se analizará la relación entre el proceso de aculturación y las actitudes sexuales de uno de los grupos minoritarios más numerosos en el país como son las personas de origen marroquí. Aunque sí se han desarrollado estudios que han analizado el proceso de aculturación en personas de origen marroquí en España (p.e., Navas et al., 2013), no lo han relacionado con las actitudes sexuales.

En cuanto a las medidas de aculturación utilizadas, los estudios que han relacionado el proceso de aculturación con las actitudes sexuales han empleado medidas indirectas (p.e., Edwards et al., 2008), unidimensionales (p.e., Huang y Uba, 1992) y bidimensionales (p.e., Brotto et al., 2005, 2007) de aculturación. En el presente estudio se utilizará una medida bidimensional siguiendo el modelo ampliado de aculturación relativa (MAAR, Navas et al., 2005). El MAAR es un modelo diseñado y adaptado a las peculiaridades de la inmigración en el contexto social y cultural de España (p.e., Navas et al., 2005, 2007), aunque se ha adaptado y utilizado en otros países como Estados Unidos (p.e., Thelamour, 2017). El MAAR distingue entre el plano ideal y el plano real de la aculturación (p.e., Navas et al., 2005). Por un lado, en el plano ideal estarían las preferencias, que hacen referencia a las opciones de aculturación que prefieren los inmigrantes en la sociedad de acogida. Por otro lado, en el plano real estarían las 
estrategias, que hacen referencia a las opciones de aculturación que ponen en práctica los inmigrantes en la sociedad de acogida. Asimismo, distingue estas preferencias y estrategias en distintos ámbitos de la realidad sociocultural, desde los más públicos hasta los más privados de la cultura (Navas y Rojas, 2010): político, bienestar social, laboral, económico, social, familiar, religioso y de valores.

De los estudios que relacionan el proceso de aculturación con las actitudes sexuales, sólo el realizado por Blanc y Rojas (2020) en Estados Unidos se apoya en el MAAR. Concretamente, incluye las preferencias de aculturación en los ámbitos más privados (social, familiar, religioso y de valores). Los ámbitos de aculturación son de especial relevancia. Por ejemplo, ámbitos más públicos (según el MAAR) como el económico (p.e., tipo de comida que consumen) podrían tener menor influencia en las actitudes sexuales que ámbitos más privados como el ámbito social (p.e., formas de relacionarse), el ámbito religioso o el de valores (p.e., igualdad entre hombres y mujeres). El estudio de Blanc y Rojas (2020), además de las preferencias en los ámbitos más privados de MAAR, también incluye indicadores de preferencias y estrategias en el ámbito sexual (tipos y formas de llevar a cabo los comportamientos sexuales, formas de expresar la sexualidad con la pareja, etc.).

En resumen, el presente estudio surge debido a que hasta la fecha ninguna investigación ha relacionado el proceso de aculturación con las actitudes sexuales en España. Por ello, el objetivo ha sido relacionar el proceso de aculturación y las actitudes sexuales (concretamente las actitudes hacia comportamientos sexuales), en uno de los grupos minoritarios más numerosos en este país como son las personas de origen marroquí. Del mismo modo que en el estudio de Blanc y Rojas (2020), realizado en Estados Unidos, para analizar el proceso de aculturación se han incluido las preferencias de aculturación en los ámbitos más privados del MAAR y las preferencias y las estrategias en el ámbito sexual. Además, en el estudio se ha controlado la variable sexo debido a que estudios anteriores (p.e., Blanc y Rojas, 2020) han encontrado que el sexo modera la relación entre el proceso de aculturación y las actitudes sexuales y el proceso de aculturación modera la relación entre el sexo y las actitudes sexuales. De acuerdo con estudios previos (p.e., Arhold y Meston, 2010; Blanc y Rojas, 2020; Brotto et al., 2005, 2007; Dang et al., 2019), se espera que las personas de origen marroquí que prefieran adoptar las costumbres de la cultura de acogida (cultura española) en los ámbitos privados del MAAR en mayor medida tengan actitudes más positivas hacia comportamientos sexuales que las personas que prefieran adoptar en menor medida dichas costumbres (Hipótesis 1). También se espera que las personas de origen marroquí que prefieran adoptar y adopten las costumbres sexuales de la cultura de acogida (cultura 
española) en mayor medida tengan actitudes más positivas hacia comportamientos sexuales que las personas que prefieran adoptar y adopten en menor medida las costumbres sexuales (Hipótesis 2).

\section{Método}

\subsection{Participantes}

Participaron 110 personas de origen marroquí (51.8\% mujeres) entre 18 y 30 años ( $M$ = 21.05; $D T=2.53$ ), seleccionadas mediante muestreo de bola de nieve. El 68.2\% ( $\mathrm{n}=$ 75) de las personas habían nacido en Marruecos y el 31.8\% ( $=35)$ habían nacido en España y sus padres en Marruecos. La media de años residiendo en España para las personas nacidas en Marruecos fue de 13.68 ( $D T=4.24)$. El 42.7\% (n = 47) tenía nacionalidad española y el 57.3\% ( $n=63$ ) nacionalidad marroquí. No se encontraron diferencias estadísticamente significativas en ninguna de las variables medidas entre las personas que habían nacido en Marruecos y las que habían nacido en España (Tabla 1).

En relación con el nivel de estudios, el 58.2\% $(n=64)$ tenía estudios secundarios, el $34.5 \%(\mathrm{n}=38)$ tenía estudios universitarios y el 7.3\% $(\mathrm{n}=8)$ tenía estudios primarios o no tenía estudios. El 96.4\% $(\mathrm{n}=106)$ señaló ser de religión musulmana y el resto ateo o agnóstico. En cuanto al estado de pareja actual, el 49.1\% ( $n=54)$ no tenía pareja, el 36.4\% $(n=40)$ tenía pareja estable y el 14.5\% $(n=16)$ tenía pareja ocasional. El 13.6\% $(n=15)$ estaba casada.

Tabla 1. Diferencias entre las personas nacidas en Marruecos y las nacidas en España en las variables medidas

\begin{tabular}{lcccc}
\hline & $\begin{array}{c}\text { Marruecos } \\
\mathrm{M}(D T)\end{array}$ & $\begin{array}{c}\text { España } \\
\mathrm{M}(D T)\end{array}$ & $t$ & $p$ \\
\hline $\begin{array}{l}\text { Pref. de aculturación ámbitos } \\
\text { privados MAAR: mantenimiento }\end{array}$ & $3.82(0.74)$ & $3.89(0.78)$ & 0.429 & .669 \\
$\begin{array}{l}\text { Pref. de aculturación ámbitos } \\
\text { privados MAAR: adopción }\end{array}$ & $3.09(0.74)$ & $3.16(0.89)$ & 0.420 & .676 \\
$\begin{array}{l}\text { Pref. de aculturación ámbito sexual: } \\
\text { mantenimiento }\end{array}$ & $3.28(1.19)$ & $3.37(1.09)$ & 0.385 & .701 \\
$\begin{array}{l}\text { Pref. de aculturación ámbito sexual: } \\
\text { adopción }\end{array}$ & $3.00(1.15)$ & $2.82(1.15)$ & -0.728 & .468 \\
$\begin{array}{l}\text { Estr. de aculturación ámbito sexual: } \\
\text { mantenimiento }\end{array}$ & $3.03(1.29)$ & $3.03(1.27)$ & 0.007 & .994 \\
$\begin{array}{l}\text { Estr. de aculturación ámbito sexual: } \\
\text { adopción }\end{array}$ & $2.83(1.14)$ & $2.66(1.33)$ & -0.688 & .493 \\
$\begin{array}{l}\text { Actitudes hacia comportamientos } \\
\text { sexuales }\end{array}$ & $56.19(16.22)$ & $54.91(18.55)$ & -0.366 & .715 \\
\hline Nota. Pref. = Preferencias; Estr. = Estrategias; MAAR = Modelo Ampliado de Aculturación Relativa
\end{tabular}




\subsection{Instrumentos}

\subsubsection{Cuestiones sociodemográficas}

Se preguntó por el sexo, la edad, el país de nacimiento, el país de nacimiento de los padres (si no había nacido en Marruecos), el nivel de estudios, la religión, el estado de pareja actual y el estado civil.

2.2.2. Escala de Actitudes hacia Comportamientos Sexuales (EACS; Blanc, Byers y Rojas, 2019)

Mide la actitud hacia comportamientos sexuales específicos en diferentes contextos. La escala está formada por 22 ítems y cada ítem hace referencia a la valoración de un comportamiento sexual. El contenido de la escala muestra comportamientos sexuales diádicos, en solitario y con más de una persona al mismo tiempo. Todos los ítems tienen un formato tipo Likert con cinco opciones de respuesta oscilando de 1 (muy negativo) a 5 (muy positivo). La puntuación total en la escala se obtiene sumando todos los ítems, pudiendo oscilar de 22 a 110. A mayor puntuación actitudes más positivas hacia los comportamientos sexuales. La fiabilidad obtenida mediante el coeficiente alfa de Cronbach en las mujeres de origen marroquí fue de .92 y en los hombres de .91.

2.2.3. Preferencias de aculturación en los ámbitos más privados según el MAAR (Navas y Rojas, 2010)

Se utilizaron dos escalas (mantenimiento y adopción) compuestas por cuatro ítems que reflejan los cuatro ámbitos más privados de aculturación (social, familiar, religioso y de valores). Una de las escalas medía la preferencia hacia el mantenimiento de la cultura de origen de la persona y la otra escala medía la preferencia hacia la adopción de la cultura de España. Las opciones de repuesta en ambas escalas oscilaban de 1 (nada) a 5 (mucho). Las puntuaciones totales de ambas escalas se obtienen promediando los ítems, pudiendo oscilar la puntuación total de 1 a 5. A mayor puntuación en la escala de mantenimiento mayor preferencia por mantener las costumbres de origen y a mayor puntuación en la escala de adopción mayor preferencia por adoptar las costumbres españolas. Para la escala de mantenimiento, la fiabilidad obtenida mediante el coeficiente alfa de Cronbach en las mujeres de origen marroquí fue de .68 y en los hombres de .77. Para la escala de adopción, la fiabilidad obtenida mediante el coeficiente alfa de Cronbach en las mujeres de origen marroquí fue de .61 y en los hombres de .69. 
2.2.4. Preferencias y estrategias de aculturación en el ámbito sexual (Blanc y Rojas, 2020)

Se realizaron dos preguntas sobre las preferencias de aculturación en el ámbito sexual. Las preguntas que se realizaron según las dimensiones de mantenimiento y adopción fueron: 1) ¿En qué grado le gusta o le gustaría mantener las costumbres sexuales (tipos y formas de llevar a cabo los comportamientos sexuales, formas de expresar la sexualidad) de Marruecos?; y, 2) ¿En qué grado le gusta o le gustaría adoptar o practicar las costumbres sexuales (tipos y formas de llevar a cabo los comportamientos sexuales, formas de expresar la sexualidad) de España?

También, se realizaron dos preguntas sobre las estrategias de aculturación en el ámbito sexual. Las preguntas que se realizaron de acuerdo con ambas dimensiones (mantenimiento y adopción) fueron: 1) ¿En qué grado mantiene las costumbres sexuales (tipos y formas de llevar a cabo los comportamientos sexuales, formas de expresar la sexualidad con la pareja, etc.) de Marruecos? y, 2) ¿En qué grado ha adoptado o practicado las costumbres sexuales (tipos y formas de llevar a cabo los comportamientos sexuales, formas de expresar la sexualidad con la pareja, etc.) de España?

Las opciones de repuesta oscilaban de 1 (nada) a 5 (mucho) en las cuatro preguntas. A mayor puntuación en las preguntas de mantenimiento mayor preferencia por mantener o mayor mantenimiento de las costumbres sexuales de origen. A mayor puntuación en las preguntas de adopción mayor preferencia por adoptar o mayor adopción de las costumbres sexuales de España.

\subsection{Procedimiento}

La aplicación del cuestionario se realizó de forma online con una duración aproximada de 10 minutos. Se identificó a una persona de origen marroquí que fue la encargada de difundir el estudio entre personas de su mismo origen. Concretamente, les proporcionó información sobre el objetivo del estudio y los criterios de inclusión. Las personas interesadas facilitaban su correo electrónico y recibían más información sobre el estudio y el enlace del cuestionario para poder participar. El estudio fue aprobado por el Comité de Bioética de Investigación Humana (CIH) de la Universidad de Almería.

\subsection{Análisis de los datos}

En primer lugar, se calcularon los estadísticos descriptivos para cada una de las 
variables medidas diferenciando entre las personas que habían nacido en Marruecos y las que habían nacido en España y se compararon sus medias mediante el estadístico de contraste $t$ de Student para muestras independientes. Posteriormente, se calcularon los estadísticos descriptivos para cada una de las variables medidas y se compararon las medias de mantenimiento y adopción cultural mediante el estadístico de contraste $t$ de Student para muestras relacionadas. También se compararon las medias en todas las medidas entre las mujeres y los hombres mediante el estadístico de contraste $t$ de Student para muestras independientes. Cuando las diferencias eran estadísticamente significativas se calculó el tamaño del efecto mediante la $d$ de Cohen $(d=$ o.1: efecto pequeño, $d=0.3$ : efecto medio y $d=0.5$ : efecto grande). Seguidamente, se correlacionaron las variables medidas mediante el coeficiente de correlación de Pearson. Para explorar si existía relación entre el proceso de aculturación y las actitudes hacia comportamientos sexuales, además de los análisis de correlación, se llevaron a cabo diferentes análisis de regresión lineal múltiple. En cada análisis se utilizó una medida de aculturación y para controlar el efecto del sexo se introdujo en cada uno de ellos la variable sexo. Esto es, se incluyeron como variables independientes (tipificadas) la medida de aculturación y la variable sexo y como variable dependiente la puntuación total en la Escala de Actitudes hacia Comportamientos Sexuales. Finalmente, se estudió si existía interacción entre la medida de aculturación y la variable sexo en las actitudes hacia comportamientos sexuales en un segundo modelo. Cuando el efecto de interacción era significativo se representó gráficamente para su interpretación. También se calculó el tamaño de efecto mediante la $f^{2}$ de Cohen $\left(f^{2}=.02\right.$ : efecto pequeño, $f^{2}=.15$ : efecto medio, $f^{2}=.35$ : efecto grande) y la potencia. Los análisis se llevaron a cabo mediante el programa estadístico SPSS versión 25 y mediante el programa GPower versión 3.1.9.

\section{Resultados}

3.1. Estadísticos descriptivos de las variables medidas, comparación entre mantenimiento y adopción cultural y diferencias entre hombres y mujeres

En la Tabla 2 se pueden observar algunos estadísticos descriptivos (mínimo, máximo, media y desviación típica) de las variables medidas. Las puntuaciones medias en preferencias de aculturación (mantenimiento y adopción) en los ámbitos más privados del MAAR revelan que las personas de origen marroquí de la muestra prefieren mantener costumbres de su cultura de origen y también adoptar costumbres de la sociedad española en cierto grado. Aunque la comparación de medias revela que prefieren mantener más que adoptar $\left(t_{(109)}=7.540, p<.001, d=0.99\right)$. 
En el ámbito sexual, los resultados también indican que prefieren mantener costumbres sexuales de su cultura de origen y adoptar costumbres sexuales de la sociedad española, prefiriendo mantener más que adoptar $\left(t_{(109)}=2.083, p=.040, d=\right.$ o.31). Asimismo los resultados ponen de manifiesto que los participantes del estudio indican mantener costumbres sexuales de su cultura de origen y adoptar las españolas en algún grado (muy cercano al punto medio, 'algo'), sin encontrase diferencias significativas en este ámbito en mantenimiento y adopción $\left(t_{(109)}=1.505, p=.135\right)$.

Cuando se comparan las medias de las medidas de aculturación entre los hombres y las mujeres de origen marroquí, se encuentran diferencias en las preferencias por mantener en los ámbitos más privados de la cultura de origen $\left(t_{(108)}=-2.298, p=.023, d\right.$ $=0.45)$, en las preferencias por adoptar las costumbres sexuales de España $\left(t_{(108)}=3.469\right.$, $p=.041, d=0.66)$ y en la adopción de dichas costumbres $\left(t_{(106.008)}=5.612, p<.001, d=\right.$ 1.06). Las mujeres de origen marroquí prefieren mantener en mayor medida $(M=4.00$, $D T=0.71)$ las costumbres de su cultura de origen en los ámbitos privados que los hombres $(M=3.67, D T=0.76)$. Sin embargo, los hombres de origen marroquí prefieren adoptar $(M=3.32, D T=1.11)$ y adoptan $(M=3.36, D T=0.94)$ en mayor medida las costumbres sexuales españolas que las mujeres $(M=2.60, D T=1.08 ; M=2.23, D T=$ 1.17, respectivamente).

Considerando que la puntuación en la escala de actitudes hacia comportamientos sexuales puede oscilar de 22 a 110, la media en las personas de origen marroquí en esta variable indica que sus actitudes hacia comportamientos sexuales son moderadamente negativas. Además, cuando se comparan las medias en esta escala entre los hombres y las mujeres se encuentran diferencias estadísticamente significativas $\left(t_{(108)}=3 \cdot 331, p=\right.$ .001, $d=0.64)$. Los hombres de origen marroquí tienen actitudes hacia comportamientos sexuales más positivas $(M=61.11, D T=16.41)$ que las mujeres $(M=$ $50.82, D T=15.98)$.

Tabla 2. Estadísticos descriptivos de las variables medidas

\begin{tabular}{|c|c|c|c|c|}
\hline & Mínimo & Máximo & $M$ & $D T$ \\
\hline $\begin{array}{l}\text { Pref. de aculturación ámbitos privados MAAR: } \\
\text { mantenimiento }\end{array}$ & 1.50 & 5.00 & 3.84 & 0.75 \\
\hline $\begin{array}{l}\text { Pref. de aculturación ámbitos privados MAAR: } \\
\text { adopción }\end{array}$ & 1.50 & 5.00 & 3.11 & 0.72 \\
\hline $\begin{array}{l}\text { Pref. de aculturación ámbito sexual: } \\
\text { mantenimiento }\end{array}$ & 1.00 & 5.00 & $3 \cdot 31$ & 1.16 \\
\hline Pref. de aculturación ámbito sexual: adopción & 1.00 & 5.00 & 2.95 & 1.15 \\
\hline $\begin{array}{l}\text { Estr. de aculturación ámbito sexual: } \\
\text { mantenimiento }\end{array}$ & 1.00 & 5.00 & 3.03 & 1.28 \\
\hline $\begin{array}{l}\text { Estr. de aculturación ámbito sexual: adopción } \\
\text { Actitudes hacia comportamientos sexuales }\end{array}$ & $\begin{array}{c}1.00 \\
22.00\end{array}$ & $\begin{array}{c}5.00 \\
106.00\end{array}$ & $\begin{array}{c}2.77 \\
55.78\end{array}$ & $\begin{array}{c}1.20 \\
16.92\end{array}$ \\
\hline
\end{tabular}


3.2. Relación entre el proceso de aculturación y las actitudes hacia comportamientos sexuales

En la Tabla 3 se muestran las correlaciones entre las variables medidas sin diferenciar entre hombres y mujeres. En general, existe correlación estadísticamente significativa y positiva entre las preferencias de adopción en los ámbitos más privados del MAAR y las actitudes hacia comportamientos sexuales. Entre las preferencias de mantenimiento en los ámbitos más privados del MAAR y las actitudes hacia comportamientos sexuales no existe correlación estadísticamente significativa.

En cuanto al ámbito sexual, existe correlación estadísticamente significativa y positiva entre la adopción (preferencias y estrategias) de las costumbres sexuales de la cultura de acogida y las actitudes hacia comportamientos sexuales. Entre las medidas de mantenimiento en el ámbito sexual y las actitudes hacia comportamientos sexuales no existe correlación estadísticamente significativa.

Tabla 3. Correlaciones entre las variables medidas

\begin{tabular}{|c|c|c|c|c|c|c|}
\hline & 2 & 3 & 4 & 5 & 6 & 7 \\
\hline $\begin{array}{l}\text { 1. Actitudes hacia comportamientos } \\
\text { sexuales }\end{array}$ & -.164 & $.274^{* * *}$ & -.104 & $.376^{* *}$ & -.173 & $.464^{* *}$ \\
\hline $\begin{array}{l}\text { 2. Pref. de aculturación MAAR: } \\
\text { mantenimiento }\end{array}$ & - & .041 & $.542^{* *}$ & -.171 & $.218^{*}$ & $-.250^{* *}$ \\
\hline $\begin{array}{l}\text { 3. Pref. de aculturación MAAR: } \\
\text { adopción }\end{array}$ & & - & -.126 & $.532^{* *}$ & -.105 & .071 \\
\hline $\begin{array}{l}\text { 4. Pref. de aculturación ámbito } \\
\text { sexual: mantenimiento }\end{array}$ & & & - & -.264 & $.323^{* *}$ & -.127 \\
\hline $\begin{array}{l}\text { 5. Pref. de aculturación ámbito } \\
\text { sexual: adopción }\end{array}$ & & & & - & .105 & $.417^{* *}$ \\
\hline $\begin{array}{l}\text { 6. Estr. de aculturación ámbito } \\
\text { sexual: mantenimiento }\end{array}$ & & & & & - & -.020 \\
\hline $\begin{array}{l}\text { 7. Estr. de aculturación ámbito } \\
\text { sexual: adopción }\end{array}$ & & & & & & - \\
\hline
\end{tabular}

En la Tabla 4 se muestran los modelos de regresión donde se analiza la relación entre las preferencias de aculturación en los ámbitos privados del MAAR y las actitudes hacia comportamientos sexuales y los posibles efectos de interacción con la variable sexo. 
Tabla 4. Modelos de regresión para analizar la relación entre las preferencias de aculturación en los ámbitos más privados del MAAR y las actitudes hacia comportamientos sexuales y los posibles efectos de interacción con la variable sexo

\begin{tabular}{lcccc}
\hline VD: Actitudes hacia comportamientos sexuales & $\mathbf{B}$ & SE B & $\boldsymbol{\beta}$ & $\mathbf{R}^{\mathbf{2}}$ \\
\hline Modelo 1 & & & & .103 \\
Constante & 55.782 & 1.542 & & \\
Pref. de aculturación MAAR: mantenimiento & -1.739 & 1.586 & -.103 & \\
Sexo & -4.789 & 1.586 & $-.283^{* *}$ & \\
\hline Modelo 2 & & & & .140 \\
Constante & 56.500 & 1.553 & & \\
Pref. de aculturación MAAR: mantenimiento & -1.976 & 1.564 & -.117 & \\
Sexo & -4.790 & 1.561 & $-.283^{* *}$ & \\
Interacción & $\mathbf{- 3 . 3 5 6}$ & $\mathbf{1 . 5 6 8}$ &.$- .193^{*}$ & \\
\hline VD: Actitudes hacia comportamientos sexuales & $\mathbf{B}$ & $\mathbf{S E ~ B}$ & $\boldsymbol{\beta}$ & $\mathbf{R}^{\mathbf{2}}$ \\
\hline Modelo 1 & & & & .156 \\
Constante & 55.782 & 1.496 & & \\
Pref. de aculturación MAAR: adopción & 4.266 & 1.507 & $.252^{* *}$ & \\
Sexo & -4.845 & 1.507 & $-.286^{* *}$ & \\
\hline Modelo 2 & & & & .211 \\
Constante & 55.484 & 1.457 & & \\
Pref. de aculturación MAAR: adopción & 4.562 & 1.468 & $.270^{* *}$ & \\
Sexo & -4.801 & 1.464 & $-.284^{* *}$ & \\
Interacción & $-\mathbf{4 . 0 1 5}$ & $\mathbf{1 . 4 7 8}$ & $-.235^{* *}$ & \\
\hline
\end{tabular}

Nota. VD = Variable dependiente; Pref. = Preferencias; MAAR = Modelo Ampliado de Aculturación Relativa; $p<.01^{* *}, p<.05^{*}$.

Los resultados muestran que existe efecto de interacción entre las preferencias (tanto de mantenimiento como de adopción) en los ámbitos privados del MAAR y el sexo en las actitudes hacia comportamientos sexuales. En la Figura 1 se muestra que las mujeres de origen marroquí que prefieren mantener en menor medida costumbres de su cultura de origen tienen actitudes hacia comportamientos sexuales más positivas que las que prefieren mantenerlas en mayor grado. Sin embargo, en los hombres no se observa esa relación. En la Figura 1 también se puede observar que mientras que en altos niveles de preferencias de mantenimiento de las costumbres de la cultura de origen hay diferencias entre hombres y mujeres en actitudes hacia comportamientos sexuales, en bajos niveles no se encuentran esas diferencias. 
Figura 1. Efectos de interacción entre las preferencias de mantenimiento en los ámbitos privados del MAAR y el sexo en las actitudes hacia comportamientos sexuales

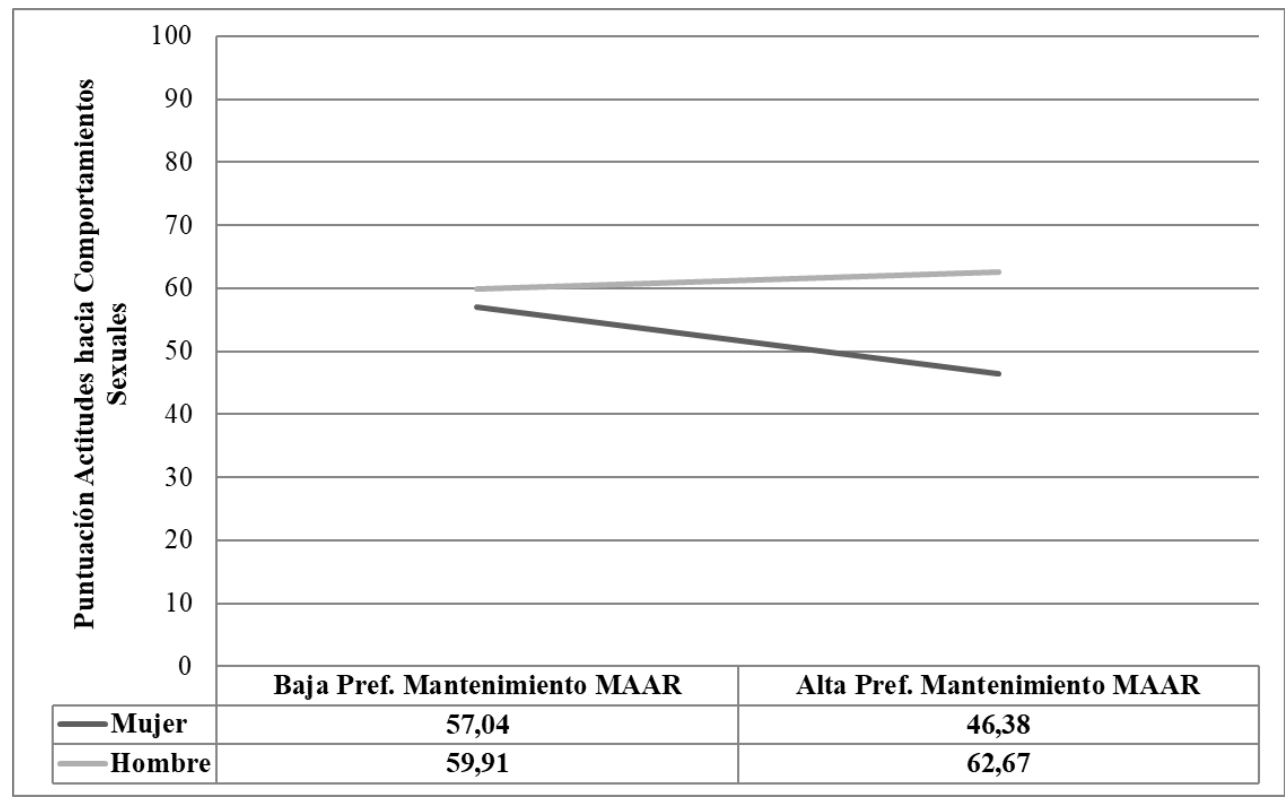

En la Figura 2 se muestra que los hombres de origen marroquí que prefieren adoptar en mayor medida costumbres españolas tienen actitudes hacia comportamientos sexuales más positivas que los que prefieren adoptarlas en menor medida. Sin embargo, en las mujeres no se observa esa relación. En la Figura 2 también se puede observar que mientras que en altos niveles de preferencias de adopción de las costumbres españolas hay diferencias entre hombres y mujeres en actitudes hacia comportamientos sexuales, en bajos niveles no se encuentran esas diferencias.

Figura 2. Efectos de interacción entre las preferencias de adopción en los ámbitos privados del MAAR y el sexo en las actitudes hacia comportamientos sexuales

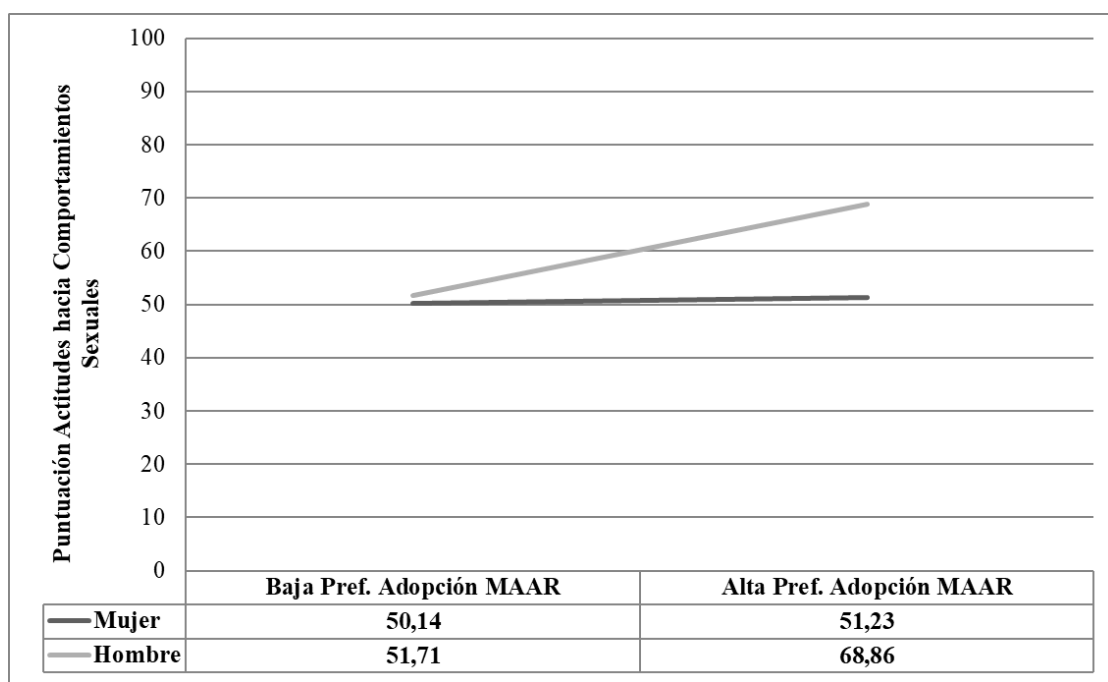

Por tanto, los resultados muestran, por un lado, que el sexo modera la relación entre 
las preferencias de mantenimiento (o adopción) cultural y las actitudes hacia comportamientos sexuales y, por otro lado, que las preferencias de mantenimiento (o adopción) cultural moderan la relación entre el sexo y las actitudes hacia comportamientos sexuales.

En la Tabla 5 se muestran los modelos de regresión donde se analiza la relación entre las preferencias de aculturación (de mantenimiento y de adopción) en el ámbito sexual y las actitudes hacia comportamientos sexuales y los posibles efectos de interacción con la variable sexo. Los resultados muestran que no existe efecto de interacción entre las preferencias en dicho ámbito y el sexo en las actitudes hacia comportamientos sexuales. Sin embargo, sí se observa que mientras que las preferencias de mantenimiento en el ámbito sexual no se relacionan con las actitudes hacia comportamientos sexuales, las preferencias de adopción en este ámbito sí lo hacen $\left(f^{2}\right.$ de Cohen $=.220$, potencia $\left.=.999\right)$. Las personas de origen marroquí que prefieren adoptar en mayor medida las costumbres sexuales de España (cultura de acogida) tienen actitudes hacia comportamientos sexuales más positivas que las que prefieren adoptar en menor medida dichas costumbres.

Tabla 5. Modelos de regresión para analizar la relación entre las preferencias de aculturación en el ámbito sexual y las actitudes hacia comportamientos sexuales y los posibles efectos de interacción con la variable sexo

\begin{tabular}{|c|c|c|c|c|}
\hline $\begin{array}{lll}\text { VD: Actitudes hacia comportamientos } \\
\text { sexuales }\end{array}$ & $\mathbf{B}$ & SE B & $\boldsymbol{\beta}$ & $\mathbf{R}^{\mathbf{2}}$ \\
\hline Modelo 1 & & & & .104 \\
\hline Constante & 55.782 & 1.542 & & \\
\hline $\begin{array}{l}\text { Pref. de aculturación ámbito sexual: } \\
\text { mantenimiento }\end{array}$ & -1.736 & 1.549 & -.103 & \\
\hline Sexo & -5.736 & 1.549 & $-.305^{* *}$ & \\
\hline Modelo 2 & & & & .106 \\
\hline Constante & 55.787 & 1.547 & & \\
\hline $\begin{array}{l}\text { Pref. de aculturación ámbito sexual: } \\
\text { mantenimiento }\end{array}$ & -1.749 & 1.554 & -.103 & \\
\hline Sexo & -5.154 & 1.554 & $-.305^{* *}$ & \\
\hline Interacción & -0.864 & 1.560 & -.051 & \\
\hline $\begin{array}{l}\text { VD: Actitudes hacia comportamientos } \\
\text { sexuales }\end{array}$ & $\mathbf{B}$ & SE B & $\boldsymbol{\beta}$ & $\mathbf{R}^{\mathbf{2}}$ \\
\hline Modelo 1 & & & & .180 \\
\hline Constante & 55.782 & 1.475 & & \\
\hline Pref. de aculturación ámbito sexual: adopción & 5.249 & 1.562 & $.310^{* *}$ & \\
\hline 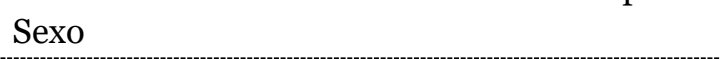 & -3.502 & 1.562 & $-.207^{* *}$ & \\
\hline Modelo 2 & & & & .180 \\
\hline Constante & $55 \cdot 762$ & 1.562 & & \\
\hline Pref. de aculturación ámbito sexual: adopción & 5.248 & 1.569 & $-310^{* *}$ & \\
\hline Sexo & -3.501 & 1.569 & $-.207^{*}$ & \\
\hline Interacción & -0.063 & 1.575 & -.004 & \\
\hline
\end{tabular}

En la Tabla 6 se muestran los modelos de regresión donde se analiza la relación entre 
las estrategias (de mantenimiento y de adopción cultural) en el ámbito sexual y las actitudes hacia comportamientos sexuales y los posibles efectos de interacción con la variable sexo. Los resultados muestran que no existe efecto de interacción entre las estrategias en dicho ámbito y el sexo en las actitudes hacia comportamientos sexuales. Sin embargo, se observa que mientras que la estrategia de mantenimiento cultural en el ámbito sexual no se relaciona con las actitudes hacia comportamientos sexuales, la estrategia de adopción cultural en este ámbito sí lo hace $\left(f^{2}\right.$ de Cohen $=.290$, potencia $=$ .999). Las personas de origen marroquí que adoptan en mayor medida las costumbres sexuales de España (cultura de acogida) tienen actitudes hacia comportamientos sexuales más positivas que las que adoptan en menor medida dichas costumbres.

Tabla 6. Modelos de regresión para analizar la relación entre las estrategias de aculturación en el ámbito sexual y las actitudes hacia comportamientos sexuales y los posibles efectos de interacción con la variable sexo

\begin{tabular}{|c|c|c|c|c|c|}
\hline $\begin{array}{l}\text { VD: Actitudes hacia } \\
\text { sexuales }\end{array}$ & comportamientos & B & SE B & $\boldsymbol{\beta}$ & $\mathbf{R}^{2}$ \\
\hline Modelo 1 & & & & & .122 \\
\hline Constante & & 55.782 & 1.525 & & \\
\hline $\begin{array}{l}\text { Estr. de aculturación } \\
\text { mantenimiento }\end{array}$ & ámbito & -2.891 & 1.532 & -.171 & \\
\hline Sexo & & -5.146 & 1.532 & $-.304^{* *}$ & \\
\hline Modelo 2 & & & & & .139 \\
\hline Constante & & 55.767 & 1.518 & & \\
\hline $\begin{array}{l}\text { Estr. de aculturación } \\
\text { mantenimiento }\end{array}$ & ámbito & -3.536 & 1.591 & $-.209^{*}$ & \\
\hline Sexo & & -5.141 & 1.525 & $-.304^{* *}$ & \\
\hline Interacción & & 2.296 & 1.615 & .134 & \\
\hline $\begin{array}{l}\text { VD: Actitudes hacia } \\
\text { sexuales }\end{array}$ & comportamientos & B & SE B & $\boldsymbol{\beta}$ & $\mathbf{R}^{2}$ \\
\hline Modelo 1 & & & & & .225 \\
\hline Constante & & 55.782 & 1.434 & & \\
\hline $\begin{array}{l}\text { Estr. de aculturación } \\
\text { adopción }\end{array}$ & ámbito & 6.960 & 1.634 & $.411^{* *}$ & \\
\hline Sexo & & -1.877 & 1.634 & -.111 & \\
\hline Modelo 2 & & & & & .228 \\
\hline Constante & & 55.296 & 1.642 & & \\
\hline $\begin{array}{l}\text { Estr. de aculturación } \\
\text { adopción }\end{array}$ & ámbito sexual: & 7.187 & 1.676 & $.425^{* *}$ & \\
\hline Sexo & & -1.732 & 1.654 & -.102 & \\
\hline Interacción & & -1.096 & 1.696 & -.056 & \\
\hline
\end{tabular}

Nota. VD = Variable dependiente; Estr. $=$ Estrategias $; p<.01^{* *}, p<.05^{*}$. 


\section{Discusión}

El fenómeno de las migraciones internacionales ha llevado a un aumento de personas de diferentes grupos etnoculturales minoritarios en las distintas sociedades, y debido al papel que posee la cultura en la sexualidad, ha cobrado interés el análisis de las actitudes sexuales de estos grupos en comparación con las del grupo mayoritario (p.e., Ahrold y Meston, 2010). Además del origen etnocultural, otra de las variables que ha sido analizada en estos contextos multiculturales ha sido el proceso de aculturación (p.e., Guo, 2019). Los estudios que han analizado la relación entre el proceso de aculturación y las actitudes sexuales se han desarrollado en Estados Unidos y Canadá. Sin embargo, en España no se ha llevado a cabo ningún estudio que analice la relación entre estas dos variables. Por ello, el objetivo del presente estudio fue relacionar el proceso de aculturación con las actitudes sexuales (concretamente las actitudes hacia comportamientos sexuales) en personas de origen marroquí. Para ello se consideró la variable sexo por su papel moderador entre estas dos variables (Blanc y Rojas, 2020).

En relación con el proceso de aculturación, en los ámbitos privados del MAAR los resultados muestran que las personas de origen marroquí prefieren mantener costumbres de su cultura de origen y adoptar costumbres de la cultura de acogida (“integración”). Aunque prefieren mantener en mayor medida las costumbres de su cultura de origen que adoptar las de la cultura de acogida (costumbres españolas). Estos resultados coindicen parcialmente con algunos de los encontrados en otro estudio realizado en España (Navas et al., 2004) con inmigrantes magrebíes (la mayoría de origen marroquí). En dicho estudio se encontró que la actitud general de aculturación manifestada era también la "integración": los inmigrantes magrebíes preferían mantener sus costumbres y al mismo tiempo adoptar las de la sociedad de acogida. Aunque, de forma más específica, se encontró que en ámbitos privados como el ámbito familiar y religioso preferían "separarse" (mantener y no adoptar). En el presente estudio, en el ámbito sexual, del mismo modo que en los ámbitos privados del MAAR, también prefieren mantener y adoptar en cierto grado. Aunque finalmente mantienen en igual medida que adoptan las costumbres sexuales.

En cuanto a las diferencias entre sexos, cuando se comparan las medidas de aculturación los resultados revelan dos aspectos relevantes. Por un lado, que las mujeres de origen marroquí prefieren mantener en mayor medida que los hombres las costumbres de origen en los ámbitos privados del MAAR. Por otro lado, que los hombres prefieren adoptar y adoptan en mayor medida que las mujeres las costumbres sexuales españolas. En cuanto a las actitudes hacia comportamientos sexuales, los hombres de 
origen marroquí presentan actitudes hacia comportamientos sexuales más positivas que las mujeres. En esta línea, Swinkels (2019) también encontró en otro país (Holanda) que los hombres de origen marroquí tenían actitudes sexuales más permisivas que las mujeres. No obstante, a pesar de que los hombres tienen actitudes hacia comportamientos sexuales más positivas que las mujeres, las actitudes de ambos grupos son más negativas que las evidencias en otros estudios con mujeres y hombres de nacionalidad española (p.e., Blanc, 2021). En el estudio de Blanc (2021), donde participaron personas de nacionalidad española, también se encontró que los hombres tienen actitudes hacia comportamientos sexuales más positivas que las mujeres.

Con respecto a la relación del proceso de aculturación en los ámbitos privados (según el MAAR) con las actitudes hacia comportamientos sexuales, se observan resultados diferentes en mujeres y hombres de origen marroquí. Por un lado, se observa que las mujeres de origen marroquí que prefieren mantener en menor medida costumbres de su cultura de origen tienen actitudes hacia comportamientos sexuales más positivas que las mujeres que prefieren mantenerlas en mayor grado. Por otro lado, como se esperaba (Hipótesis 1) se observa que los hombres de origen marroquí que prefieren adoptar en mayor medida costumbres españolas tienen actitudes hacia comportamientos sexuales más positivas que los hombres que prefieren adoptarlas en menor grado. Estos resultados muestran como el sexo modera la relación entre el proceso de aculturación y las actitudes sexuales, siendo coherentes con los encontrados en otros estudios realizados en otros países (Ahrold y Meston, 2010; Blanc y Rojas, 2020). Blanc y Rojas (2020) también encontraron que existía relación entre la adopción de las costumbres de la cultura dominante y las actitudes hacia comportamientos sexuales en hombres africanos/afroamericanos en Estados Unidos, pero no en las mujeres del mismo grupo etnocultural. Igualmente, en línea con los resultados de este estudio, Ahrold y Meston (2010) encontraron que existía relación entre el mantenimiento de las costumbres de la cultura de origen y determinado tipo de actitudes sexuales (roles tradicionales de género) en mujeres asiáticas e hispanas en Estados Unidos, pero no en hombres de los mismos grupos etnoculturales.

Los resultados también muestran que el proceso de aculturación en los ámbitos privados (según el MAAR) modera la relación entre el sexo y las actitudes sexuales. Mientras que en altos niveles de preferencias de mantenimiento de las costumbres de la cultura de origen y en altos niveles de preferencias de adopción de las costumbres españolas hay diferencias entre hombres y mujeres en actitudes hacia comportamientos sexuales, en bajo niveles no se encuentran esas diferencias. Blanc y Rojas (2020) también hallaron diferencias entre hombres y mujeres latinos/hispanos $y$ 
africanos/afroamericanos en altos niveles de mantenimiento y no en bajos niveles de mantenimiento. Aunque, en contraste con este estudio, en un grupo etnocultural (asiáticos) hallaron diferencias entre mujeres y hombres en las actitudes hacia comportamientos sexuales en bajos nivel de adopción de las costumbres de la cultura dominante y no en altos nivel de adopción. Esto demuestra, como en otros estudios (Ahrold y Meston, 2010; Blanc y Rojas, 2020), que las relaciones entre el proceso de aculturación y las actitudes sexuales no son iguales en todos los grupos (papel moderador del origen etnocultural).

Finalmente, como se esperaba (Hipótesis 2), los resultados del presente estudio apuntan en la misma dirección que los encontrados en estudios realizados en otros países con muestras diferentes (p.e., Arhold y Meston, 2010; Blanc y Rojas, 2020; Brotto et al., 2005, 2007; Dang et al., 2019). Las personas de origen marroquí que prefieren adoptar en mayor medida las costumbres sexuales de la cultura de España (cultura de acogida) y que aseguran adoptarlas en mayor medida, tienen actitudes hacia comportamientos sexuales más positivas que las que prefieren adoptar y las adoptan en menor medida. Sin embargo, en la dimensión de mantenimiento de las costumbres sexuales (tanto para las preferencias como para las estrategias), cuando se controla el sexo, no se encuentra relación con las actitudes hacia comportamientos sexuales. Como se apuntó en la introducción, los resultados de los estudios que relacionan el mantenimiento de las costumbres de la cultura de acogida y las actitudes sexuales no son consistentes. En este estudio sólo encontramos relación entre el mantenimiento de la cultura de origen en los ámbitos privados del MAAR y las actitudes sexuales en las mujeres. Arhold y Meston (2010) encontraron relación con algunas medidas específicas de actitudes sexuales, y Blanc y Rojas (2020) en algunos grupos etnoculturales. Sin embargo, otros estudios no han encontrado relación entre el mantenimiento de la cultura de origen y las actitudes sexuales (p.e., Brotto et al., 2007; Woo et al., 2011).

Finalmente, hay que destacar que los resultados de este estudio apoyan la importancia de incluir el proceso de aculturación cuando se estudian las actitudes hacia comportamientos sexuales de las personas de origen marroquí en España. Aunque en dichas personas predominan unas actitudes sexuales, la interacción con otros grupos podría conducir a la modificación de sus actitudes. En el presente estudio se observa cómo la adopción de las costumbres sexuales de la sociedad española se encuentra relacionada con unas actitudes hacia comportamientos sexuales más positivas. No obstante, los resultados de este estudio se deben interpretar teniendo en cuenta algunas limitaciones. Entre dichas limitaciones se encontraría el tamaño de la muestra y el tipo de muestreo empleado. Debido a la dificultad de accesibilidad de la muestra se utilizó un 
muestreo no aleatorio. También hay que matizar que algunos de los coeficientes de fiabilidad estimados en las escalas de aculturación son relativamente bajos. Por todo ello, en futuras investigaciones se debería ampliar la muestra y llevar a cabo un estudio en mayor profundidad de las propiedades psicométricas de las escalas de aculturación en jóvenes de origen marroquí. Por otro lado, en futuros estudios se podría relacionar el proceso de aculturación y las actitudes sexuales en otros grupos etnoculturales minoritarios con alta presencia en España, como son las personas de origen rumano o ecuatoriano. Podría ser que en estos grupos etnoculturales la relación entre el proceso de aculturación y las actitudes sexuales fuera diferente.

A pesar de las limitaciones encontradas, a través de este estudio se pone de manifiesto que, en un contexto multicultural como España, se debe considerar no sólo el origen etnocultural (además de otras variables como el sexo), sino también el proceso de aculturación cuando se analizan las actitudes hacia comportamientos sexuales de las personas de origen marroquí. El proceso de aculturación no sólo está relacionado directamente con las actitudes hacia comportamientos sexuales, sino que modera la relación entre el sexo y dichas actitudes. Por tanto, a través de este estudio se evidencia que las actitudes sexuales de las personas de origen marroquí pueden comprenderse mejor examinando el papel que juega la aculturación. Además, debido a que las actitudes sexuales también se relacionan con la salud sexual (Woo et al., 2011), sería conveniente en los programas de prevención e intervención en salud sexual con personas de origen marroquí impulsar aquella estrategia de aculturación que promueva una excelente salud sexual. 


\section{Referencias}

Abdolsalehi-Najafi, E. y Beckman, L. J. (2013). Sex guilt and life satisfaction in IranianAmerican women. Archives of Sexual Behavior, 42, 1063-1071. doi: 10.1007/s10508-013-0084-2

Ahrold, T. K. y Meston, C. M. (2010). Ethnic differences in sexual attitudes of U.S. college students: Gender, acculturation, and religiosity factors. Archives of Sexual Behavior, 39, 190-202. doi: 10.1007/s10508-008-9406-1

Berry, J. W. (2003). Conceptual approaches to acculturation. En K. M. Chun, P. B. Organista y G. Marín (Eds.), Acculturation. Advances in theory, measurement, and applied research (pp. 17-37). Washington, DC: American Psychological Association.

Blanc, A. (2021). El papel mediador de las actitudes entre los comportamientos sexuales de hombres y mujeres. Revista Internacional de Andrología, 19, 107-111. doi: 10.1016/j.androl.2019.11.002

Blanc, A., Byers, S. E. y Rojas A. J. (2019). Attitudes toward Sexual Behaviors Scale. En R. R. Milhasuen, J. D. Sakaluk, C. M. Davis y W. L. Yarber (Eds.), Handbook of sexuality-related measures (4th ed.) (pp. 423-426). New York: Routledge.

Blanc, A. y Rojas, A. J. (2020). Attitudes toward sexual behaviours in different ethnocultural groups and their relationship with the acculturation process. Ceskoslovenska Psychologie, 3, 342-359.

Brotto, L. A., Chik, H. M., Ryder, A. G., Gorzalka, B. B. y Seal, B. N. (2005). Acculturation and sexual function in Asian women. Archives of Sexual Behavior, 34, 613-626. doi: 10.1007/s10508-005-7909-6

Brotto, L. A., Woo, J. S. y Ryder, A. G. (2007). Acculturation and sexual function in Canadian East Asian men. The Journal of Sexual Medicine, 4, 72-82. doi: 10.1111/j.1743-6109.2006.00388.x

Castañeda, D. M. (2017). Sexting and sexuality in romantic relationships among Latina/o emerging adults. American Journal of Sexuality Education, 12, 120-135. doi: 10.1080/15546128.2017.1298069

Dang, S. S., Hewitt, P. L. y Brotto, L. A. (2019). Heritage and mainstream acculturation's association with sexual response in young Chinese men and women in Canada. The Canadian Journal of Human Sexuality, 28, 159-176. doi: 10.3138/cjhs.20190017

Du, H. y Li, X. (2015). Acculturation and HIV-related sexual behaviours among international migrants: A systematic review and meta-analysis. Health Psychology Review, 9, 103-122. doi: 10.1080/17437199.2013.840952

Edwards, L. M., Fehring, R. J., Jarrett, K. M. y Haglund, K. A. (2008). The influence of 
religiosity, gender, and language preference acculturation on sexual activity among Latino/a adolescents. Hispanic Journal of Behavioral Sciences, 30, 447462. doi: 10.1177/0739986308322912

Eisenman, R. y Dantzker, M. L. (2006). Gender and ethnic differences in sexual attitudes at a Hispanic-serving university. Journal of General Psychology, 133, 153-162. doi: 10.3200/GENP.133.2.153-162

Fugère, M. A., Escoto, C., Cousins, A. J., Riggs, M. L. y Haerich, P. (2008). Sexual attitudes and double standards: A literature review focusing on participant gender and ethnic background. Sexuality and Culture, 12, 169-182. doi: 10.1007/s12119-008-9029-7

Guo, Y. (2019). Sexual double standards in White and Asian Americans: Ethnicity, gender, and acculturation. Sexuality \& Culture, 23, 57-95. doi: 10.1007/s12119018-9543-1

Heinemann, J., Atallah, S. y Rosenbaum, T. (2016). The impact of culture and ethnicity on sexuality and sexual function. Current Sexual Health Reports, 8, 144-150. doi: 10.1007/s11930-016-0088-8

Huang, K. y Uba, L. (1992). Premarital sexual behavior among Chinese college students in the United State. Archives of Sexual Behavior, 21, 227-240. doi: 10.1007/BFo1542994

Navas, M. S., García, M. C., Sánchez, J., Rojas, A. J., Pumares, P. y Fernández, J. S. (2005). Relative Acculturation Extended Model: New contributions with regard to the study of acculturation. International Journal of Intercultural Relations, 29, 21-37. doi: 10.1016/j.ijintrel.2005.04.001

Navas, M., López-Rodríguez, L. y Cuadrado, I. (2013). Mantenimiento y adaptación cultural de diferentes grupos inmigrantes: variables predictoras. Anales de Psicología, 29, 207-216. doi: 10.6018/analesps.29.1.135491

Navas, M. S., Pumares, P., Sánchez, J., García, M. C., Rojas, A. J., Cuadrado, I., ...Fernández, J. S. (2004). Estrategias y actitudes de aculturación: la perspectiva de los inmigrantes y de los autóctonos en Almería. Sevilla: Dirección general de coordinación de políticas migratorias. Junta de Andalucía.

Navas, M. S. y Rojas, A. J. (2010). Aplicación del modelo ampliado de aculturación relativa a nuevos colectivos de inmigrantes en Andalucía: rumanos y ecuatorianos. Sevilla: Dirección general de coordinación de políticas migratorias. Junta de Andalucía.

Navas, M. S., Rojas, A. J., García, M. y Pumares, P. (2007). Acculturation strategies and attitudes according to the relative acculturation extended model (RAEM): The perspectives of natives versus immigrants. International Journal of Intercultural 
Relations, 31, 67-86. doi: 10.1016/j.ijintrel.2006.08.002

Swinkels, E. H. (2019). Casual sex and the sexual self-image of adolescents and young adults [Tesis de maestría no publicada]. Universidad de Utrecht.

Thelamour, B. (2017). Applying the Relative Acculturation Extended Model to examine Black Americans' perspectives on African immigrant acculturation. Journal of Cross-Cultural Psychology, 48, 1457-1471. doi: 10.1177/0022022117730614

Woo, J. S., Brotto, L. A. y Gorzalka, B. B. (2011). The role of sex guilt in the relationship between culture and women's sexual desire. Archives of Sexual Behavior, 40, 385-394. doi: 10.1007/s10508-010-9609-0 